\title{
IDENTIFICACIÓN DE EUSTONGYLIDES TUBIFEX (NITZCH IN RUDOLPHI, 1819) (NEMATODA: DIOCTOPHYMATIDAE) EN UNA GARZA ESTRIADA (BUTORIDES STRIATA, PELECANIFORME: ARDEIDAE) EN EL HUMEDAL EL BANCO, MICHOACÁN, MÉXICO
}

\author{
IDENTIFICATION OF EUSTRONGYLIDES TUBIFEX (NEMATODA: DIOCTOPHYMATIDAE) \\ IN A GREEN-BACKED HERON (BUTORIDES STRIATA, PELECANIFORME: ARDEIDAE) \\ IN THE WETLAND EL BANCO, MICHOACAN, MEXICO
}

\section{ALICIA E. ARRONA-RIVERA, Xóchitl HERNÁNDEZ-VELASCO,* ENRIQUE BASURTO-ARGUETA}

\author{
Departamento de Medicina y Zootecnia de Aves, Facultad de Medicina Veterinaria y Zootecnia (FMVZ), \\ Universidad Nacional Autónoma de México (UNAM). Av. Universidad 3000, Ciudad Universitaria, Del. \\ Coyoacán, CP. 04510, Ciudad de México, México. \\ * Autor de correspondencia: <xochilt_h@yahoo.com>. \\ Recibido: 01/03/2016; aceptado: 28/02/2017 \\ Editor responsable: Ricardo Rodríguez Estrella.
}

Arrona-Rivera, A. E., Hernández-Velasco, X., \& Basurto-Argueta, E. (2017). Identificación de Eustongylides tubifex (Nitzch in Rudolphi, 1819) (Nematoda: Dioctophymatidae) en una garza estriada (Butorides striata, Pelecaniforme: Ardeidae) en el humedal El Banco, Michoacán, México. Acta Zoológica Mexicana (n.s.), 33(2), 181-187.

RESUMEN. El presente trabajo reporta el hallazgo de Eustongylides tubifex en una garza estriada (Butorides striata), hembra, adulta, que fue encontrada muerta en el humedal El Banco, en el estado de Michoacán, México. A la necropsia, se observaron estructuras tubulares que formaban túneles en las paredes del proventrículo y ventrículo, y adherencias en el peritoneo.

Palabras clave: Butorides striata, eustrongiloidosis, Eustongylides tubifex, garza estriada, Michoacán, México.

\section{INTRODUCCIÓN}

La eustrongiloidosis o peritonitis verminosa, es una enfermedad parasitaria causada por nemátodos del género Eustrongylides que afecta a muchas especies animales en diversas regiones del mundo (Paperna 1974; Moravec et al. 2003; Sattari et al. 2007), y que también puede ser una zoonosis (Barros et al. 2007; Meneguetti et al. 2013). En las aves, Eustrongylides afecta principalmente a las aves piscívoras y se caracteriza por penetrar y formar túneles tortuosos en la pared del proventrículo y ventrículo, además causa adherencias en el intestino (Measures 1988a,b) y provocar alta mortalidad, principalmente en polluelos (Wiese et al. 1977; Spalding et al. 1994). La infección es por vía digestiva. El ciclo de vida del género Eustrongyli-
Arrona-Rivera, A. E., Hernández-Velasco, X., \& Basurto-Argueta, E. (2017). Identification of Eustrongylides tubifex (Nematoda: Dioctophymatidae) in a green-backed heron (Butorides striata, Pelecaniforme: Ardeidae) in the wetland El Banco, Michoacan, Mexico. Acta Zoológica Mexicana (n.s.), 33(2), 181-187.

ABSTRACT. The current report addresses the finding of Eustongylides tubifex in a green-backed heron (Butorides striata), female, adult, found dead in the wetland El banco, in the state of Michoacan, Mexico. At necropsy, tubular structures forming tunnels in the walls of the proventriculus and ventricle, and numerous adhesions in the peritoneum were observed.

Key words: Butorides striata, eustrongiloidosis, Eustongylides tubifex, green-backed heron, Michoacan, Mexico.

des presenta cuatro estadios larvales (L1 a L4) y requiere de dos hospederos intermediarios, el primero es un oligoqueto y el segundo un pez. Algunos peces depredadores, que ingieren los peces infectados, pueden ser huéspedes paraténicos (Fried \& Franson 2001). Sin embargo, también algunos anfibios y reptiles pueden actuar como hospederos intermediarios secundarios o paraténicos (Fried \& Franson, 2001).

Se han reportado tres especies de Eustrongylides que afectan aves: E. excisus (Jägerskiöld, 1909), E. ignotus (Jägerskiöld, 1909) y E. tubifex (Nitzch in Rudolphi, 1819), estas especies han sido encontradas en Gaviiformes, Anseriformes, Ciconiiformes, Pelecaniformes, Charadriiformes y Podicipediformes (Asakawa et al., 1997; Fried \& Franson, 2001); E. ignotus ha sido reportada en 
los Estados Unidos de América (Spalding et al., 1993), China (Xiong et al., 2009), Ucrania (Syrota \& Kharchenko et al., 2015); E. excisus se ha reportado en Europa y Asia (Fried \& Franson, 2001); mientras que E. tubifex ha sido reportado en Canadá (Measures, 1988b), los Estados Unidos de América (Spalding et al., 1993), Argentina (Brugni \& Viozzi, 2003), Europa, Rusia (Franson \& Custer, 1994) y Japón (Asakawa et al., 1997).

El presente trabajo reporta la presencia de E. tubifex en un ave acuática conocida como garza estriada o garza azulada (Butorides striata, Pelecaniformes: Ardeidae), y como sinónimo Butorides virescens (BirdLife International, 2014). La garza estriada tiene una distribución muy amplia, con más de 30 subespecies alrededor del mundo que poseen variaciones en el color de su plumaje, lo que en ocasiones causa confusión para identificarla dentro de la misma especie (Hancock, 1999), se le encuentra en el sureste de Canadá, Noreste de los Estados Unidos de América, en México -excepto en las tierras altas centrales del norte- (Peterson \& Chalif, 1989); así como en América Central y Sudamérica, África, Arabia y Asia (BirdLife International, 2014), en una gran variedad de hábitats acuáticos entre los cuales se encuentran riberas boscosas como costas, estuarios y manglares, bordes de ríos, lagos, canales y salinas (Hancock \& Kushlan, 1984; Kushlan \& Hancock, 2005), marismas, lagunas (Preston, 1998) marismas de hierba, pastos, campos de arroz y otros cultivos inundados (del Hoyo et al., 1992), zonas pantanosas con bordes acuosos, pequeños estanques; rara vez en áreas abierta (Howell \& Webb, 1995).

La garza azulada realiza cacerías de pie y de espera o acecho lento (Howell \& Webb, 1995). Su dieta es muy variada, se alimentan de peces (del Hoyo et al., 1992; Kushlan \& Hancock, 2005); anfibios, como ranas (del Hoyo et al., 1992); insectos, como escarabajos de agua, saltamontes y libélulas (del Hoyo et al., 1992; Kushlan \& Hancock, 2005); arañas; sanguijuelas; crustáceos, como langostinos (Hancock, 1999), cangrejos y gambas; moluscos (del Hoyo et al., 1992); lombrices de tierra y gusanos poliquetos (Kushlan \& Hancock, 2005); pequeños reptiles y ratones (del Hoyo et al., 1992).

\section{MATERIAL Y MÉTODOS}

Una garza estriada, hembra adulta sin cabeza, fue encontrada muerta en el humedal El Banco, ubicado en el Ejido Epifanio C. Pérez, Municipio de Cuitzeo del Porvenir, en el Estado de Michoacán, durante la actividad cinegética de patos silvestres migratorios de la temporada 2008 a 2009. El ejemplar fue empaquetado en una bolsa de plástico con cierre hermético, y en bolsas amarillas para residuos biológicos-infecciosos y se transportó en una hielera (licencia de colector científico SGPA/DGVS/05688/06) al Departamento de Medicina y Zootecnia de Aves (DMZA) de la Facultad de Medicina Veterinaria y Zootecnia (FMVZ) de la Universidad Nacional Autónoma de México (UNAM), para realizarle la necropsia. Al hacer la disección del estómago del ave y de los túneles en la serosa del mismo, se encontraron nemátodos de color beige, rosado o rojizo. Los parásitos fueron colectados con pinzas en un frasco con etanol al 70\% para su conservación (Doster \& Goater, 1997) y fueron enviados al laboratorio de parasitología del DMZA donde fueron aclarados en lactofenol e identificados de acuerdo con Measures (1988 a,b); además, se midió el largo y ancho de 50 huevos.

\section{RESULTADOS}

A la inspección externa, la condición corporal del ave se clasificó como 1 en la escala 1 a 5 debido a que el esternón se observó muy prominente lo cual se relacionó con un bajo desarrollo de los músculos pectorales por una mala condición nutricional (Harrison \& Ritchie, 1994). En la evaluación de órganos internos, los pulmones y el corazón presentaban hemorragias. El hígado tenía una coloración amarilla clara en todo el parénquima. Se observaron estructuras tubulares en la cavidad celómica, adherencias y exudado fibrinoso en el peritoneo (Fig. 1), y túneles en las paredes del proventrículo y ventrículo (Fig. 2). El diagnóstico morfológico macroscópico fue peritonitis fibrinosa parasitaria crónica difusa grave.

Se lograron colectar dos hembras, tres machos y segmentos de los nemátodos. Los parásitos se identificaron como Eustrongylides tubifex (Nematoda: Dioctophymatidae), de acuerdo con las características observadas en los especímenes y reportes previos (Cram, 1927; Measures, 1988b; Fastzkie \& Crites, 1977). Esta especie no posee labios, la boca es pequeña y angosta, a su alrededor posee 6 papilas internas, muy pequeñas, y 6 externas, de mayor tamaño, con un anillo nervioso que se presenta poco después de que inicia el esófago (en promedio a $235 \mu$ del extremo anterior), el cual es simple. Los nemátodos son más anchos en la parte media que en los extremos, tienen cutícula con estriaciones transversales, que se aprecia más ancha y clara en los extremos (Fig. 3). Los machos midieron en promedio $76.2 \mathrm{~mm}$ de largo x $923 \mu$ de ancho 


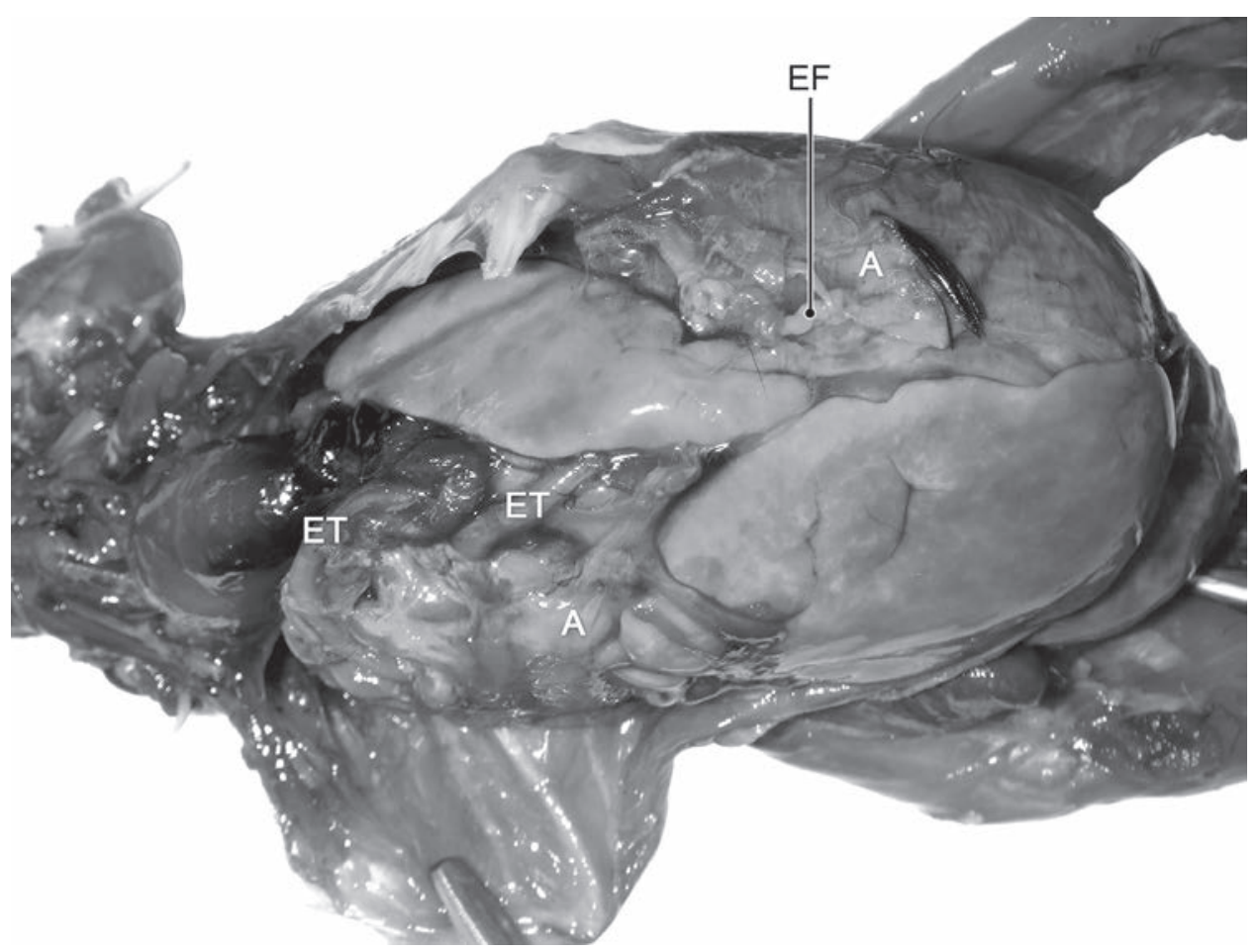

Figura 1. Cavidad celómica de una garza estriada (Butorides striata) donde se aprecia exudado fibrinoso (EF), adherencias (A), así como la presencia de estructuras tubulares (ET) en peritoneo y palidez hepática.

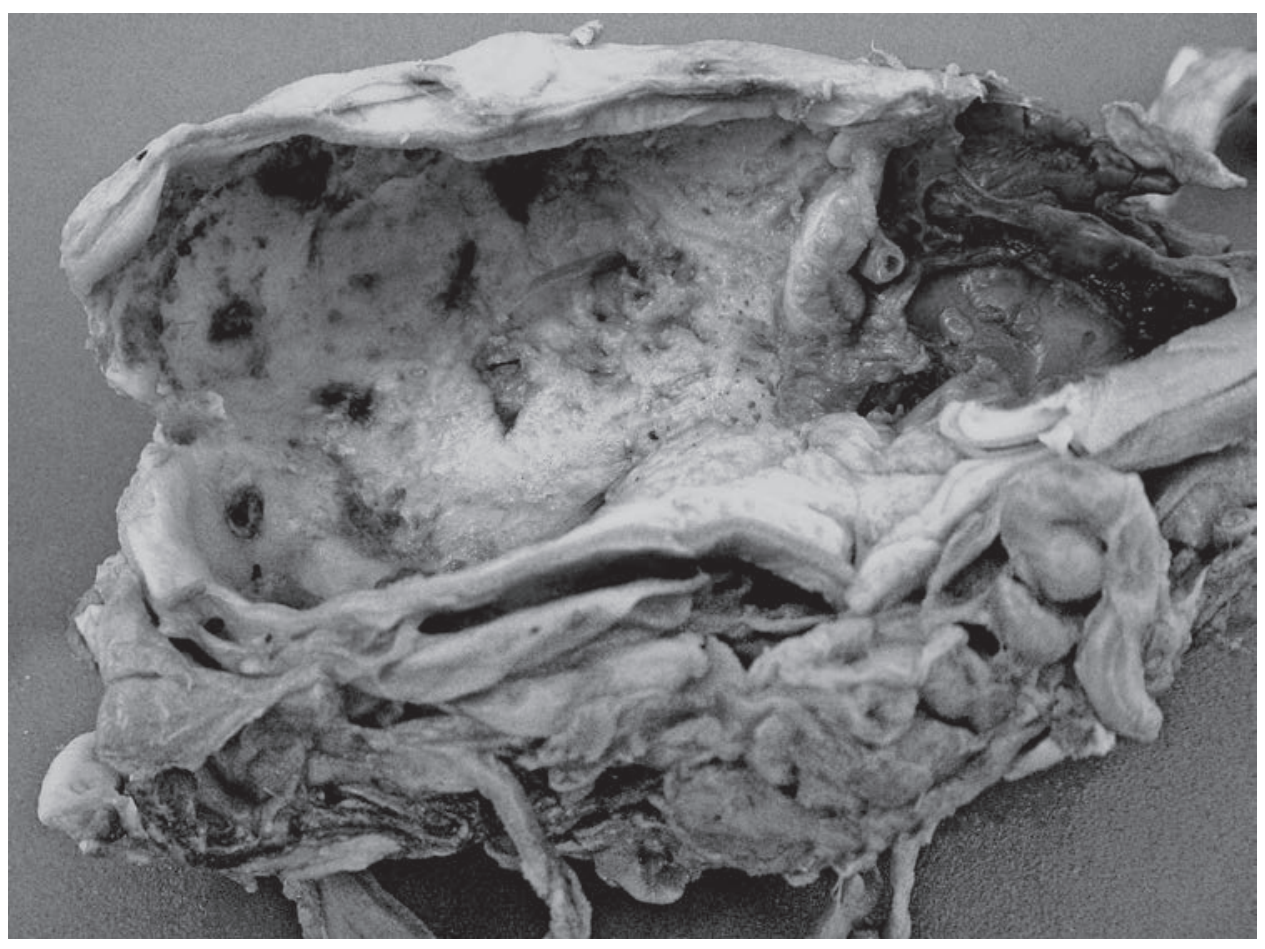

Figura 2. Vista interna del proventrículo y ventrículo de una garza estriada (Butorides striata) con múltiples túneles creados a causa de la infestación por el E. tubifex. 


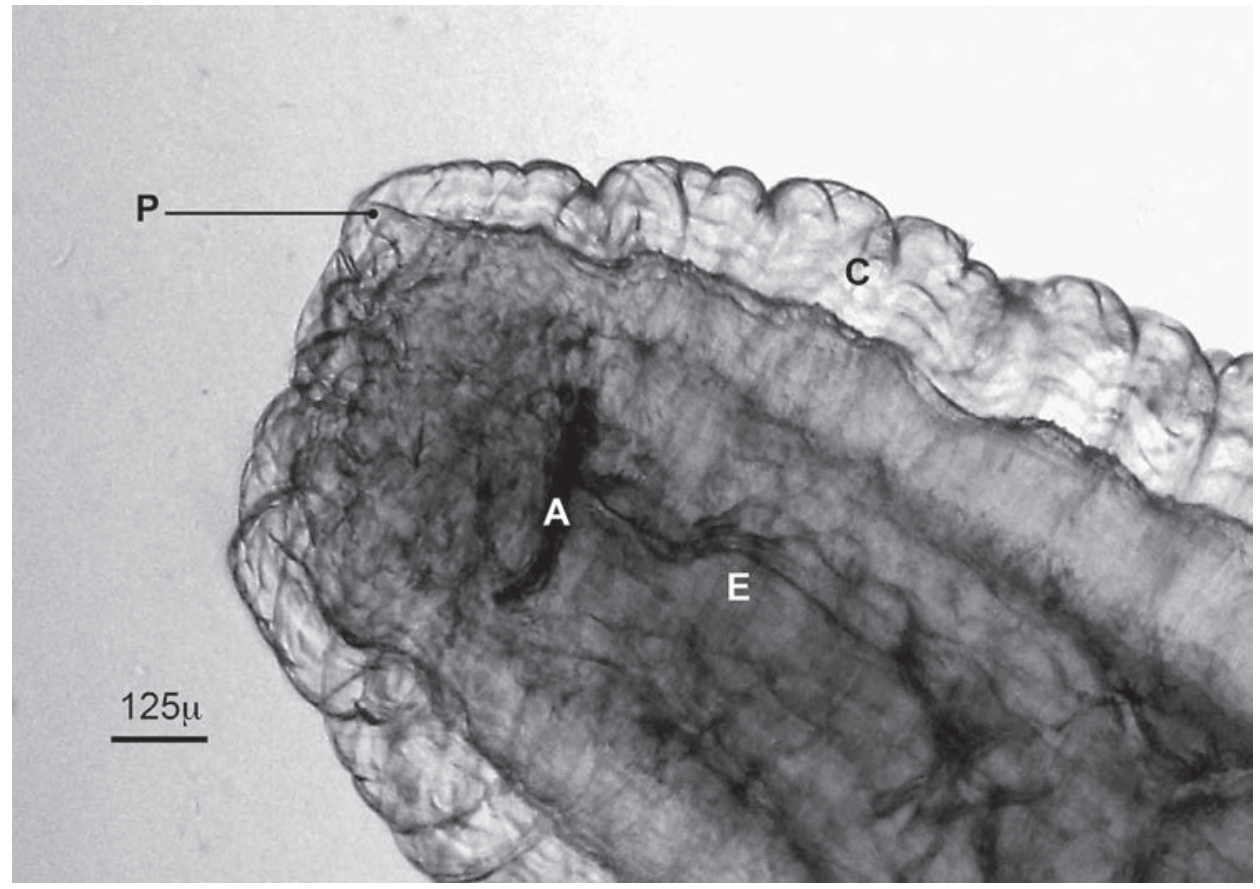

Figura 3. Vista lateral del extremo anterior de una hembra adulta de Eustrongylides tubifex, donde se aprecian la cutícula gruesa y estriada (C), las papilas externas (P), el anillo nervioso (A) y el esófago (E).

en la parte media, presentan una ventosa bursal en forma de campana (ancho promedio $407 \mu$ ) y una espícula larga que termina en punta y midió en promedio $8.9 \mathrm{~mm}$. Las hembras midieron $92 \mathrm{~mm}$ de largo x $2.87 \mathrm{~mm}$ de ancho en promedio y en su extremidad posterior cuenta con una vulva ventral (Fig. 4).

Sus huevos son ovalados, de color ámbar, con pared gruesa, de superficie irregular con múltiples depresiones, que midieron $(\mathrm{n}=50)$ entre 65 a $72.5 \mu$ de largo x 37.5 a $42 \mu$ de ancho (Fig. 5); lo cual concuerda con lo reportado por Cram (1927) y Measures (1988b), quienes menciona que los huevos de Eustrongylides tubifex miden de 69 a $77.5 \mu$ de largo x 39 a $40 \mu$ de ancho.

\section{DISCUSIÓN}

Aunque Eustrongylides ha sido reportado como un parásito común de peces y anfibios en México (Pérez-Ponce de León et al., 2000; Ramírez \& Osorio, 2002; SalgadoMaldonado, 2006), no ha sido posible la identificación a nivel de especies, debido a que estos taxa han sido aislados de hospederos intermediarios. El ciclo reproductivo de Eustrongylides consta de 4 estadios larvarios, el primero se desarrolla en el huevo, el segundo y tercero en oligoquetos (Measures, 1988c; Coyner et al., 2003) y el cuarto en peces y ocasionalmente en anfibios (Ramírez \& Osorio, 2002). Los hospederos definitivos son las aves ictiófagas que consumen peces infectados (Spalding \& Forrester, 1993; Ramírez \& Osorio, 2002).

La veloz construcción de canales y la residencia en ellos, fuera del tracto digestivo, pero en contacto con él, es el mecanismo que Eustrongylides utiliza para obtener alimento, para expulsar sus huevecillos junto con las heces del ave, y para evitar ser expulsado del huésped, debido a que no cuenta con estructuras para fijarse a las paredes del tracto digestivo, como otros parásitos (Spalding \& Forrester, 1993). En este caso, durante la inspección del cadáver estaba emaciado y a la necropsia se observaron las superficies de los órganos en la cavidad celómica inflamados, cubiertos de fibrina, con adherencias y una gran cantidad de túneles largos y tortuosos en la superficie del proventrículo y ventrículo, lo cual concuerda con lesiones y hallazgos reportados previamente como característicos de Eustrongylides (Spalding, 1990; Spalding \& Forrester, 1993; Fried \& Franson, 2001).

Eustrongylides no sólo afecta la salud de las aves ictiófagas, sino también sus poblaciones, causa alta mortalidad, principalmente en polluelos (Wiese et al., 1977; Spalding et al., 1993, 1994). La muerte se relaciona con 


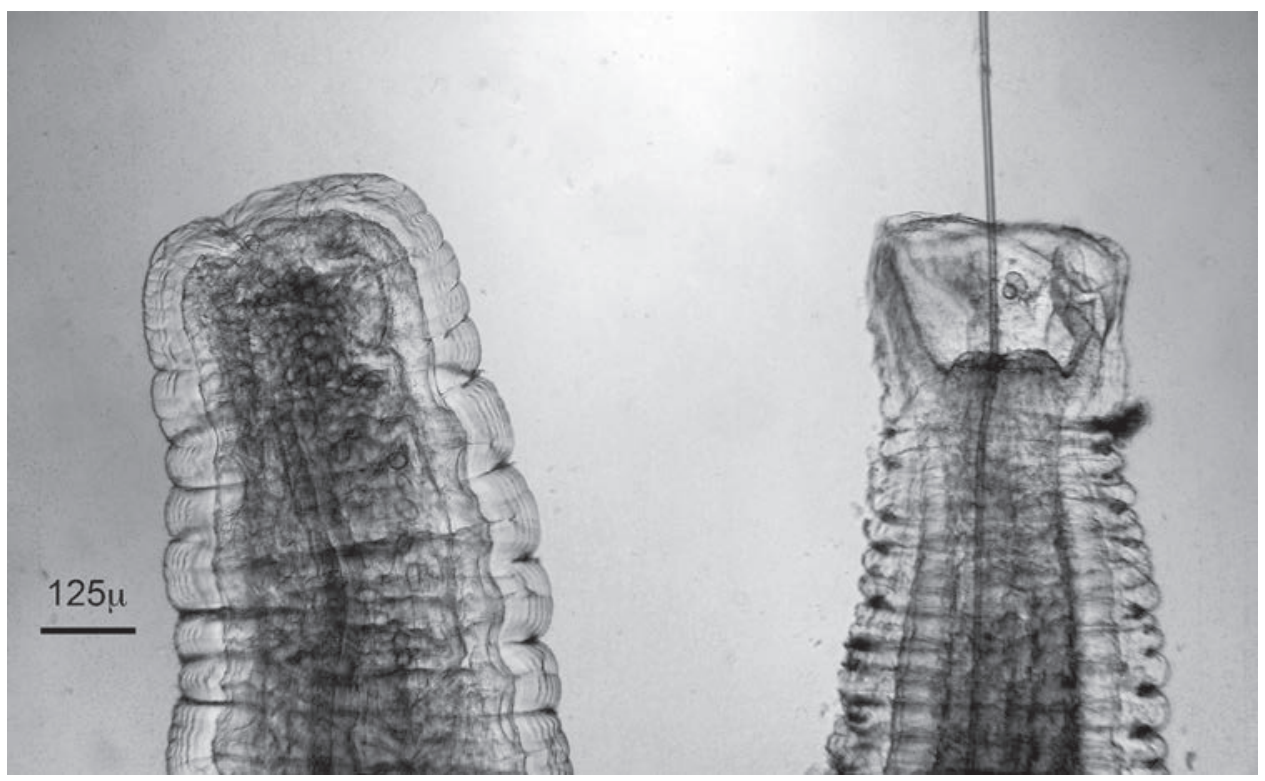

Figuras 4. Eustrongylides tubifex adultos. Extremidad caudal de una hembra (izquierda) donde se aprecia la vulva terminal con huevos, y de un macho (derecha) donde se observa la bursa y espícula características.

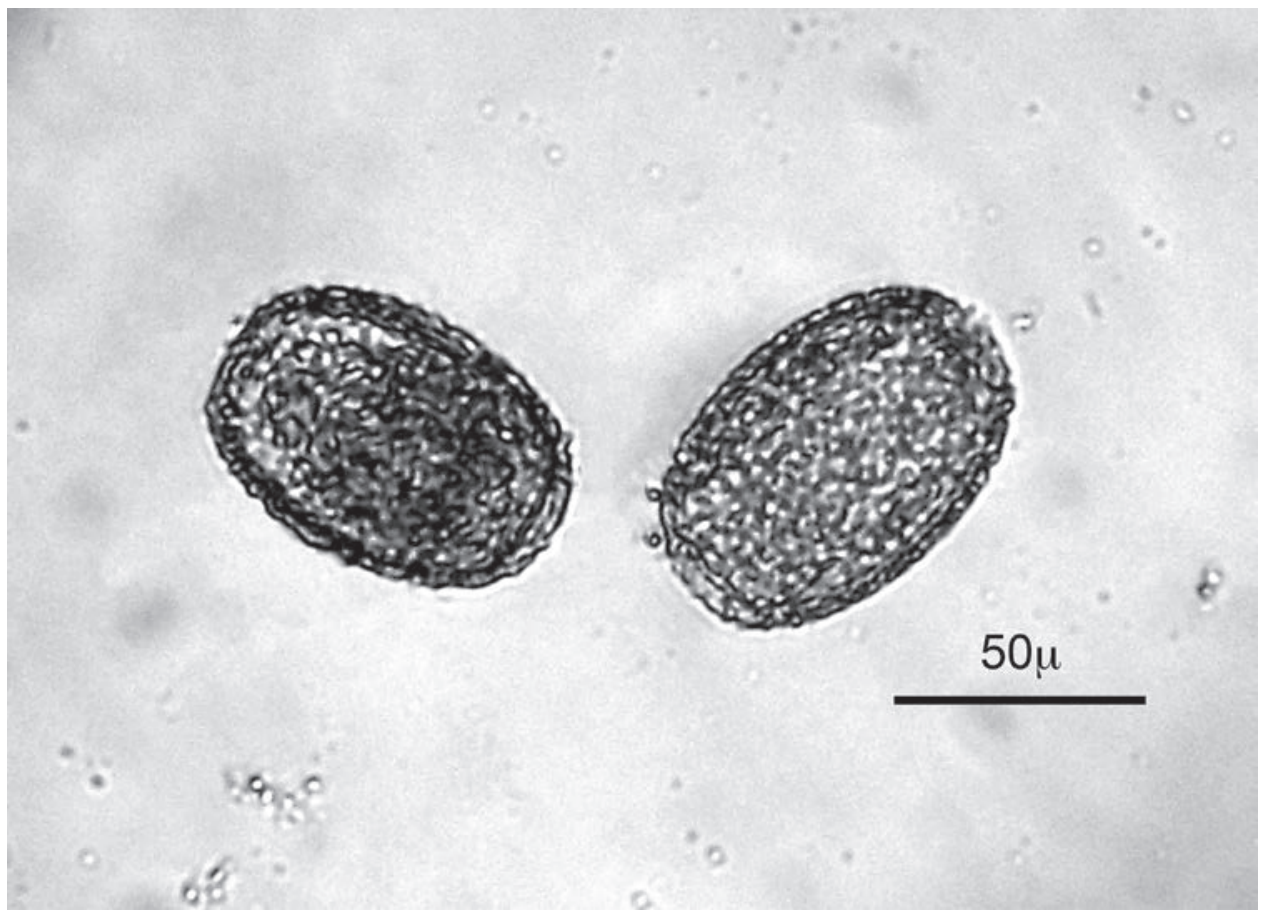

Figura 5. Huevos de E. tubifex donde se observa su pared gruesa y con múltiples depresiones.

la cantidad de parásitos (Spalding et al., 1993; Spalding \& Forrester, 1993), y al nivel de daño que causa en los tejidos de las aves (Locke et al., 1964; Roffe, 1988), como peritonitis, emaciación, fallas orgánicas u obstrucción intestinal (Fried \& Franson, 2001). De acuerdo con Spalding et al., (1993) las aves jóvenes son más susceptibles a la infección parasitaria por Eustrongylides y a medida que aumenta la edad del ave se requiere una mayor can- 
tidad de parásitos para causarle la muerte. En este caso la garza estriada fue encontrada muerta y sin cabeza, por lo que no podemos saber si la infestación por Eustrongylides contribuyó a su muerte; sin embargo, a la necropsia se encontró una peritonitis crónica difusa severa causada por este parásito y que se relacionó con la mala condición corporal del ave.

En México no existen estudios epidemiológicos que muestren la prevalencia de la eustrongiloidosis en aves ictiófagas, los aspectos que influyen en las interacciones con sus hospederos, ni el efecto de esta infección en las poblaciones, migración y reproducción de las aves a las que afecta.

El registro de la presencia de una especie del género Eustrongylides en el humedal El Banco, en el estado de Michoacán reviste importancia, debido a que no existen reportes previos de eustrongyloidosis en garza estriada (Butorides striata) en México; y desde el punto de vista ecológico y zoosanitario, ya que este parásito puede causar alta mortalidad en polluelos de aves ictiófagas y reducir sus poblaciones (Wiese et al., 1977; Spalding et al., 1994), e incrementar del riesgo de transmisión al hombre a través del consumo de pescados o ranas crudos o mal cocidos (Spalding \& Forrester, 1993; Ramírez \& Osorio, 2002). Además, Eustrongylides ha sido considerado como un indicador de contaminación ambiental por eutrofización de las aguas (Spalding \& Forrester, 1993; Fried \& Franson, 2001). Sin embargo, son necesarios estudios que analicen las interacciones entre nematodos parásitos y aves ictiófagas; una cooperación más estrecha entre investigadores de diversos campos de estudio, que permitan conocer los efectos de estos parásitos en las poblaciones de aves piscívoras acuáticas y de litoral de México; así como, analizar si existe relación entre la descarga de aguas negras en los humedales y lagos con un incremento de la población de oligoquetos y con la prevalencia de Eustrongylides en los peces y en las aves que se alimentan de ellos.

AGRADECIMIENTOS. Los autores agradecen los comentarios y sugerencias realizados por los revisores anónimos.

\section{LITERATURA CITADA}

Asakawa, M., Kimoto, Y. \& Murata, K. (1997). First record of Eustrongylides tubifex (Dioctophymatidae) from Little Grebe, Tachybaptus ruficollis in Japan. The Journal of Veterinay Medical Science, 59, 955-956.

Barros, L. A., Moraes Filho, J. \& Oliveira, R. L. (2007). Larvas de nematóides de importância zoonótica encontradas em traíras (Ho- plias malabaricus bloch, 1794) no município de Santo Antonio do Leverger, M. T. Arquivo Brasileiro de Medicina Veterinária e Zootecnia, 59, 533-535.

BirdLife International.(2014). Butorides striata. The IUCN Red List of Threatened Species 2014: e.T22728182A40831320. http://dx.doi. org/10.2305/IUCN.UK.2014-2.RLTS.T22728182A40831320.en

Brugni, N. \& Viozzi, G. (2003). Presencia de Eustrongylides tubifex (Nematoda: Dioctophymatoidea) en la Patagonia, Argentina. $\mathrm{Pa}$ rasitología Latinoamericana, 58, 83-85.

Coyner, D. F., Spalding, M. G. \& Forrester D. J. (2003). Epizootiology of Eustrongylides ignotus in Florida: transmission and development of larvae in intermediate hosts. Journal of Parasitoogy, 89, 290-298.

Cram, E. B. (1927.) Bird parasites of the nematode subordens Strongylata, Ascaridata and Spiruarata. Bulletin of the United States National Museum, 140, 367-375.

del Hoyo, J., Elliot, A. \& Sargatal, J. (1992). Handbook of the birds of the world, vol. 1: ostrich to ducks. Lynx Editions, Barcelona, Spain, 696 pp.

del Hoyo, J., Collar, N. J., Christie, D. A., Elliott, A. \& Fishpool, L. D. C. (2014). HBW and BirdLifeInternational illustrated checklist of the birds of the world. Barcelona, Spain and Cambridge UK, Lynx Editions and BirdLife International, 904 pp.

Doster, G. L. \& Goater, C. P. (1997). Collection and quantification of avian helminths and protozoa. Pp 396-418. In: D. H. Clayton \& J. Mooru (Eds.). Host-parasite evolution. Oxford University Press, New York, USA.

Fastzkie, J. S. \& Crites, J. L. (1977). A Redescription of Eustrongylides tubifex (Nitzsch 1819) Jägerskiöld 1909 (Nematoda: Dioctophymatidae) from Mallards (Anas platyrhynchos). The Journal of Parasitology, 63, 707-712.

Franson, J. C. \& Custer, T. W. (1994). Prevalence of eustrongylidosis in wading birds from colonies in California, Texas, and Rhode Island, United States of America. Colonial Waterbirds, 17, 168-172.

Fried, J. M. \& Franson, J. C. (2001). Eustrongylidosis. Pp. 223-228. In: Field manual of wildlife disease: birds. US Department of the interior \& U S Geological Survey. Madison WI, USA.

Hancock, J. \& Kushlan, J. (1984). The herons handbook. Croom Helm, London, 288 pp.

Hancock, J. (1999). Herons and egrets of the world. Academic Press, London, UK, 208 pp.

Harrison, G. J. \& Ritchie, B. (1994). Making distinctions in the physical examination, pp. 144-175. In: B. Ritchie, G. J. Harrison \& L. R. Harrison (Eds.). Avian medicine: Principles and applications. Wingers Publishing Inc., Florida, EUA.

Howell, S. N. G. \& Webb, S. A. (1995). Guide to the birds of Mexico and Northern Central America. Oxford University Press, Oxford, NJ, 1010 pp.

Kushlan, J. A. \& Hancock, J. A. (2005). The herons. Oxford University Press, Oxford, U.K.

Locke, L. N., De Withh, J. B., Menzie, C.M., Kerwin, J. A. (1964). A merganser die-off associated with larval Eustrongylides. Avian Disease, 8, 420-427.

Measures, L. N. (1988a). Revision of the genus Eustrongylides Jägerskihöld, 1909 (Nematoda: Dioctophymatoidea) of piscivorous bird. Canadian Journal of Zoology, 66, 845-895. 
Measures, L. N. (1988b). Epizootiology, pathology and description of Eustrongylides tubifex (Nematoda: Dioctophymatoidea) in fish: Canadian Journal of Zoology, 66, 2212-2222.

Measures, L. N. (1988c). The development and pathogenesis of Eustrongylides tubifex (Nematoda: Dioctophymatoidea) in oligochaetes. Journal of Parasitology 74, 294-304.

Meneguetti, D. U., Laray, M. P. \& Camargo, L. M. A. (2013). Primeiro relato de larvas de Eustrongylides sp. (Nematoda: Dioctophymatidae) em Hoplias malabaricus (Characiformes: Erythrinidae) no Estado de Rondônia, Amazônia Ocidental, Brasil. Revista Pan-Amazônica de Saúde, 4, 55-58.

Moravec, F., Nie, P. \& Wang, G. T. (2003). Some nematodes of fishes from central China, with the redescription of Procamallanus (Spirocamallanus) fulvidraconis (Camallanidae). Folia Parasitologica, 50, 220-230.

Paperna, I. (1974). Hosts distribution and pathology of infections with larvae of Eustrongylides (Dioctophymidae, Nematoda) in fish from East African lakes. Journal of Fish Biology, 6, 67-76.

Pérez-Ponce de León, G., García-Prieto, L., León-Règagnon, V. \& Choudhury, A. (2000). Helminth communities of native and introduced freshwater fishes of Lake Pátzcuaro, Michoacán, México. Journal of Fish Biology, 57, 303-325.

Peterson, R. T. \& Chalif, E. L. (1989). Aves de México, guía de campo de todas las especies encontradas en México, Guatemala, Belice y el Salvador. Diana, DF, México, 473 pp.

Preston, E. E. (1998). A field guide to the birds of Mexico and adjacent areas: Belize, Guatemala, and El Salvador. University of Texas Press, Austin, TX, 292 pp.

Ramírez, L. J. \& Osorio, S. D. (2002). Lesiones histológicas en músculo esquelético causadas por larvas de Eustrongylides sp. (Nematoda: Dioctophymatidae) de ranas comestibles del Lago Cuitzeo, Michoacán, México. Veterinaria México, 33, 335-341.
Roffe, T. J. (1988). Eustrongylides sp. Epizootic in young common egrets (Casmerodius albus). Avian Diseases, 32, 143-147.

Salgado-Maldonado, G. (2006). Checklist of helminth parasites of freshwater fishes from Mexico. Zootaxa, 1324, 1-354.

Sattari, M., Mokhayer, B., Khara, H., Nezami, S. \& Shafii, S. (2007). Occurrence and intensity of parasites in some bonyfish species of Anzali wetland from the southwest of the Caspian Sea. Bulletin of the European Association of Fish Pathologists, 27, 54-60.

Spalding, M. G. (1990). Antemortem diagnosis of eustrongylidosis in wading birds (Ciconiiformes) Colonial Waterbirds, 13, 7577.

Spalding, M. G. \& Forrester D. J. (1993). Pathogenesis of Eustrongylides ignotus (Nematoda: Dioctophymatoidea) in Ciconiiformes. Journal of Wildlife Diseases, 29, 250-260.

Spalding, M. G., Bancroft, G. T. \& Forrester, D. J. (1993). The Epizootiology of eustrongylidosis in wading birds (Ciconiiformes) in Florida. Journal of Wildlife Diseases, 29, 237-249.

Spalding, M. G., Smith J. P. \& Forrester D. J. (1994). Natural and experimental infections of Eustrongylides ignotus: Effect on growth and survival of nestling wading birds. The Auk, 111, 328336.

Syrota, Y.Y. \& Kharchenko, V. O. (2015). Analysis of study comprehensiveness for nematode fauna of hydrophilic birds in Ukrainian Polissya. Annals of Parasitology, 61, 165-174.

Wiese, J. H., Davidson, W. R., \& Nettles, V. F. (1977). Large scale mortality of nestling ardeids caused by nematode infection: Journal of Wildlife Diseases, 13, 376-382.

Xiong, F., Wang, G. T., Wu, S. G., \& Nie, P. (2009). Development of Eustrongylides ignotus (Nematoda: Dioctophmida) in domestic ducks (Anas platyrhynchos domestica L.). Journal of Parasitology, 95, 1035-1039. 